\title{
Present status of the 4-m ILMT data reduction pipeline: application to space debris detection and characterization
}

\author{
Bikram Pradhan $^{1 *}$, Ludovic Delchambre ${ }^{1}$, Paul Hickson ${ }^{2}$, \\ Talat Akhunov ${ }^{3}$, Przemyslaw Bartczak ${ }^{4}$, Brajesh Kumar ${ }^{5}$, Jean Surdej ${ }^{1}$
}

${ }^{1}$ Space sciences, Technologies and Astrophysics Research (STAR) Institute,

Université de Liège, Allée du 6 Août 19c, 4000 Liège, Belgium

2 Department of Physics and Astronomy, The University of British Columbia, 6224 Agricultural Road, Vancouver, BC, V6T1Z1, Canada

${ }^{3}$ Ulugh Beg Astronomical Institute of the Uzbek Academy of Sciences, Astronomicheskaya 33, 100052 Tashkent, Uzbekistan

${ }^{4}$ Poznań Observatory, Adam Mickiewicz University, Sloneczna 36, 60-101 Poznań, Poland

${ }^{5}$ Indian Institute of Astrophysics, Koramangala, Bangalore 560 034, India

\begin{abstract}
The 4-m International Liquid Mirror Telescope (ILMT) located at the ARIES Observatory (Devasthal, India) has been designed to scan at a latitude of $+29^{\circ} 22^{\prime} 26^{\prime \prime}$ a band of sky having a width of about half a degree in the Time Delayed Integration (TDI) mode. Therefore, a special data-reduction and analysis pipeline to process online the large amount of optical data being produced has been dedicated to it. This requirement has led to the development of the 4-m ILMT data reduction pipeline, a new software package built with Python in order to simplify a large number of tasks aimed at the reduction of the acquired TDI images. This software provides astronomers with specially designed data reduction functions, astrometry and photometry calibration tools. In this paper we discuss the various reduction and calibration steps followed to reduce TDI images obtained in May 2015 with the Devasthal $1.3 \mathrm{~m}$ telescope. We report here the detection and characterization of nine space debris present in the TDI frames.
\end{abstract}

\section{Introduction}

The 4-m International Liquid Mirror Telescope (ILMT) will continuously scan a $\sim 0.5^{\circ}$ band of sky in the Time Delayed Integration (TDI) mode, also known as drift scan, where the telescope cannot be tilted and hence cannot track the way conventional telescopes do (Borra, Hickson and Surdej 2009, Surdej et al. 2006). In this particular technique, the CCD detector tracks by electronically stepping its pixels and gigabytes of information will be stored on disk during each night of observation. This

\footnotetext{
*E-mail : bpradhan@ulg.ac.be
} 
huge amount of data must be reduced and analyzed in a timely fashion and it is clearly necessary to automatize as much as possible these operations in order to increase the speed at which they can be carried out. The existing general-purpose astronomical software packages are not sufficiently efficient to perform such tasks. The 4-m ILMT data reduction pipeline has been designed specifically to carry out the reduction of the TDI data and to perform their astrometric and photometric calibrations.

In order to prepare ourselves for when the ILMT will become operational, a replica of direct ILMT observations has been carried out using the ARIES $1.3 \mathrm{~m}$ optical telescope at Devasthal, India. In this paper, we present the optimal algorithms adopted to reduce these science images. An interesting application of this software is to contribute to the statistical awareness on space environment around the Earth. In this context, we present the detection and characterization of 9 space debris. Most of these appear during the TDI observations carried out at dawn and dusk.

\section{Science data reduction}

Each image obtained with the Devasthal $1.3 \mathrm{~m}$ telescope (May 2015) equipped with a direct CCD camera $(2 \mathrm{~K} \times 2 \mathrm{~K})$ working in the TDI mode consists of a long strip of sky covering a field of view of nearly $2.3^{\circ}$ in right ascension and $0.15^{\circ}$ in declination. The data reduction procedure includes the following steps: dark subtraction, flat-field correction, cosmic ray removal, sky subtraction, astrometric and photometric calibration.

In our case the science images show a systematic variation in the gradient of the dark current along the declination direction as a function of the changing ambient temperature during the night. To correct for this effect, we have first calculated the average of the pixels along the right ascension axis for each dark frame so that we obtain a 1 dimensional $2 \mathrm{~K}(2048)$ file of pixel values along the declination axis. The plot shown in Fig. 1 illustrates the different slopes derived of a straight line fit to the above mentioned pixel values for different temperatures. It is clearly evident that there is a change in the slope of the straight lines as a function of the CCD temperature. So, we have developed appropriate algorithms to subtract at best the relevant dark current.

In the dark subtracted science images, the average of the pixel values along the right ascension axis with sigma-clipping is taken in order to only include those pixels enlightened by the sky so that we avoid all pixels illuminated by stars, cosmic rays and exclude the effects of bad pixels. We get 2048 pixel values along the declination axis which can be treated as a 1D flat. Since the star images are trailed along the rows of the CCD, the pixel sensitivity issue becomes a row (1D) sensitivity one. And the pixel-non-uniformity is corrected by dividing the dark subtracted frame by the normalized 1D flat (as shown in Fig. 2) throughout all the columns. An example of CCD frame before and after the flat field correction is illustrated in Fig. 3.

During a continuous and long TDI observation, the sky may vary throughout the night due to the presence of the moon, clouds, dusk light and/or twilight. The numerical approach implemented in the sky background estimation consists of an optimized combination of "sliding average method" and "two dimensional polynomial fit" along the right ascension axis which shows the trend in variation of the sky level as time goes. The variation of the sky brightness during a single night and the estimated sky value are shown in Fig. 4.

After dark subtraction, flat field correction and sky subtraction, our Python module is capable of calibrating all sources located within the band of sky covering about $2.3^{\circ}$ in right ascension and $0.15^{\circ}$ in declination with a precision of $\sim 0.3^{\prime \prime}$. Then the aperture photometry technique has been used to derive the magnitude of the detected celestial objects (Mighell 1999). As the observation has been carried out at zenith with least air-mass and best seeing conditions, the magnitude of the detected sources are derived after comparing the instrumental and apparent magnitudes of a set of photometric standard stars (see Fig. 5). 


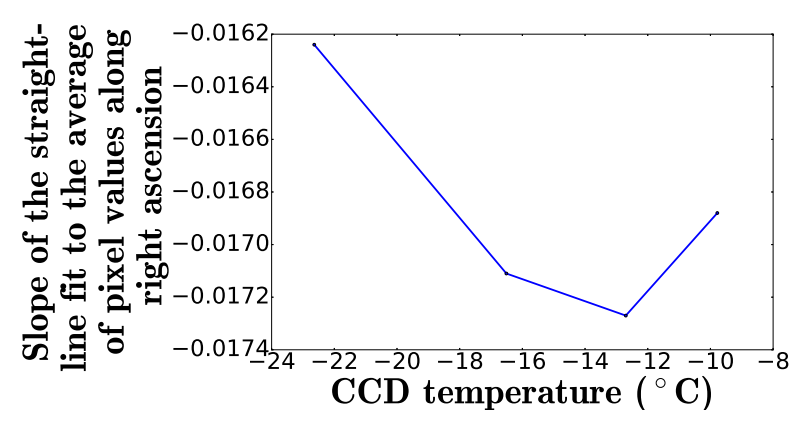

Figure 1: Varying behavior of the dark current as a function of the CCD temperature.
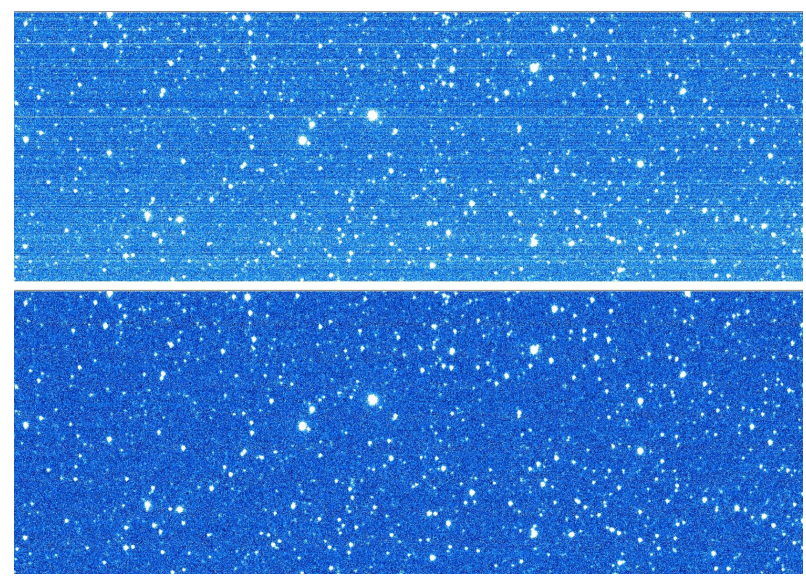

Figure 3: The upper and lower images show an example of CCD frame before and after flat field correction, respectively

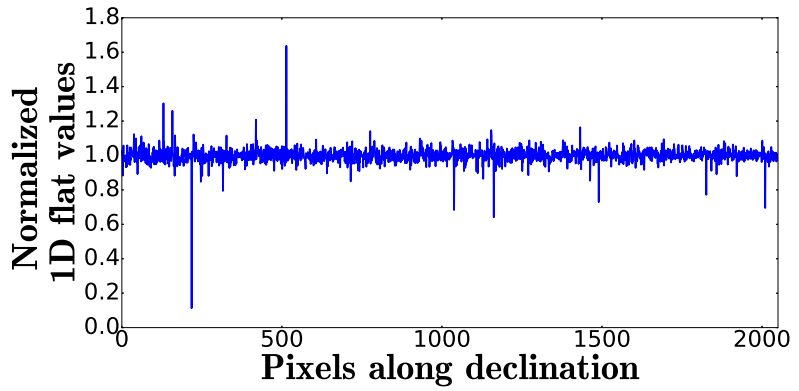

Figure 2: One dimensional normalized flat field obtained from the dark-subtracted pixel values along the right ascension axis after sigmaclipping.

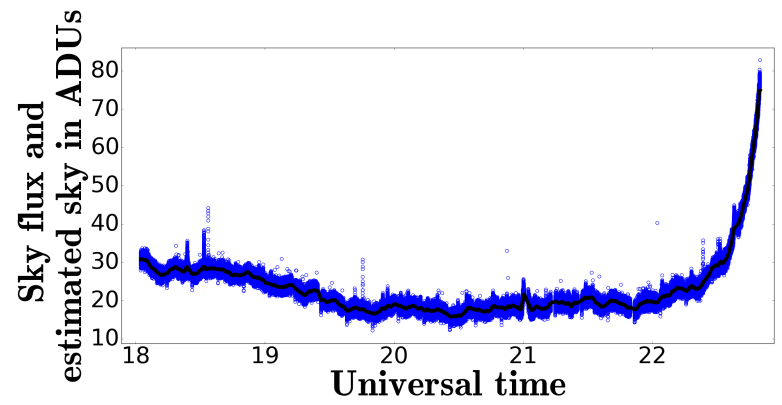

Figure 4: Variation of the sky background as a function of time during a single night of observation. The plot in blue shows the sky flux in ADU and the thick black line shows the estimated sky level, also in ADU.

\section{Debris detection and identification}

We have detected a total of 12 space debris during the TDI observations obtained during the nights from 16 until 26 of May 2015 using the ARIES Devasthal 1.3m telescope. A collage of detected space debris is shown in Fig. 6. In total, 9 debris out of 12 have been identified using a software developed at the University of British Columbia (Canada). The software is a Python module that predicts the positions and other characteristics of space debris that pass near the zenith at a particular time using a two line element (TLE).

\section{Future prospects}

Later on the data reduction pipeline will be integrated into the real time observations performed with the ILMT. Photometric calibration will be improved using multiple PSF fitting in crowded stellar fields. The performance of our pipeline will be significantly improved with parallel computations using high-performance processors and servers. Identified space debris will be characterized in terms of their magnitude and period of rotation from the recorded light curves. 


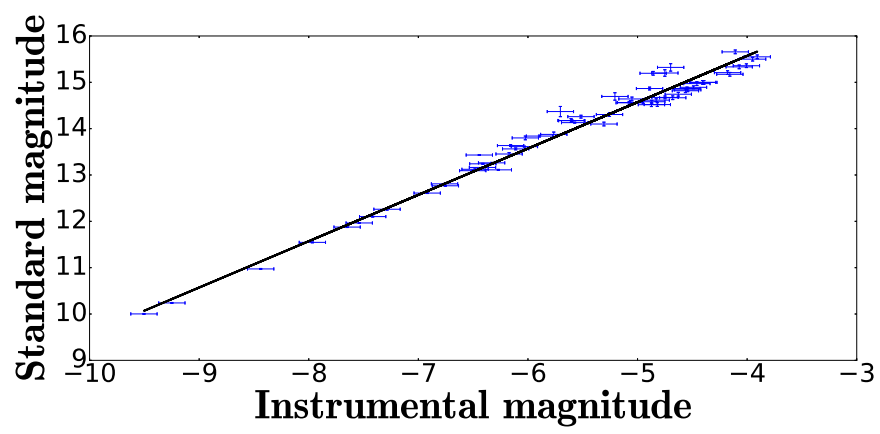

Figure 5: Photometric calibration: the calibrated magnitude of each source can be found using the linear relation established between the instrumental and standard magnitudes.

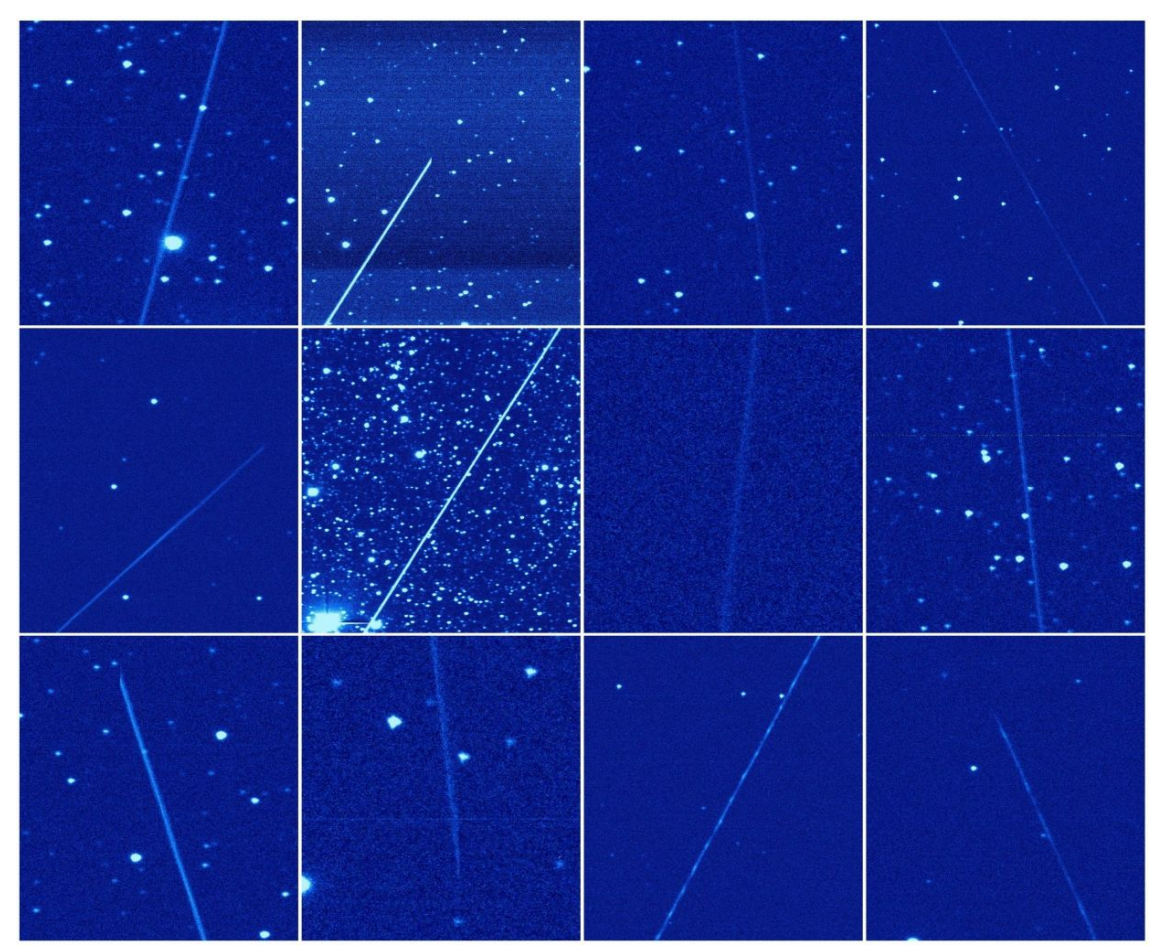

Figure 6: Collage of 12 detected space debris

\section{Acknowledgements}

This research has been partially supported by the 'Programme de bourse d'excellence IN.WBI (Belgium)', Région Wallonne (Belgium) under the convention 516238, F.R.S.-FNRS (Belgium), the Liège University and the Natural Sciences and Engineering Research Council of Canada.

\section{References}

Borra E., Hickson P., Surdej J. 2009, OptPN, 20, 28

Surdej J. , Absil O., Bartczak P. et al. 2006, Proceedings of the SPIE conference, Orlando, Vol. 6267, 626704

Mighell, K. J. 1999, in ASP Conf. Ser., Vol. 172, Astronomical Data Analysis Software and Systems VII 